\title{
Aspects of methodology of pedagogics in an informational society
}

\author{
Alibek H. Dzamyhov ${ }^{1}$, Magomedhan M. Nimatulaev ${ }^{2, *}$, and Petr Yur. Romanov ${ }^{3}$ \\ ${ }^{1}$ Karachai-Cherkess state University, Department of mathematics and methods of its teaching; Russia \\ ${ }^{2}$ Financial University under the Government of the Russian Federation, Department of Business Informatics, Moscow, Russia \\ ${ }^{3}$ Magnitogorsk state technical University, Department of Mathematics, Magnitogorsk, Russia
}

\begin{abstract}
In this article, the contents of methodology of pedagogics and the problems of its representation in modern education are considered. The system-information methodological approach and its realization in formal, content-based, social and cultural and other methodological approaches to education are described.
\end{abstract}

\begin{abstract}
"Methodology derives from "method" and from Greek "word", "concept", "doctrine" is a system of principles and means of organization and formation of theoretical and practical activities, as well as the doctrine of this system" [1]. "Logy" in this compound word, as in similar "philology", "psychology", indicates the logical motivation and the structure of "the doctrine", presence of systemacy and logic models of its structure and the contents. The main component of the word "methodology" is "method". Therefore, method is the main topic of this "doctrine" (in the broadest sense), its contents, logical and semantic representation. The doctrine - the rationale, the organization is dedicated to it.
\end{abstract}

The general method in the cognitive system, «method in its broad sense is understood as the main general trend, complete strategy» [2]. According to I.L.Bim, it is a generalized model of realization of the principles of knowledge united by a certain dominating idea applied to its general aims, which shows how and where it is necessary to move to achieve it. Therefore, the main and determining components of knowledge is the system of methodological approaches and principles, as well as the system of their purposeful realization. We will consider methodology of pedagogics in this aspect.

It is well known that the highest level of methodology of knowledge is the philosophical knowledge. General scientific approaches and principles, applicable to all spheres of knowledge are required too.

Researchers in each differential science, including, perhaps, first of all, pedagogics, should not only possess necessary philosophical knowledge, but must also be able to develop it, solving the methodological tasks. Hence, it is necessary to incorporate methodology of knowledge into one logical unit, one system. "Methodology is an independent science», [3]. Besides, since the methodology science has nonvacuous intersection with each differential science it has its

\footnotetext{
* Corresponding author: tim@instrao.ru
}

representation in a scientific metasystem (system of systems), corresponding to the metasystem of all science.

Thus, methodology of pedagogics is the scientific and pedagogical system having projections in didactics of each educational subject. That is, it is a pedagogical metasystem. The objective methodology is a scientifically methodical system which is the description, the content of methodological system in didactics of this subject.

The world and the society change, they become informational, get informational representation. Science as well as its methodology is also evolving, turning more and more informational. Hence, general scientific and main approaches, knowledge and research principles are changing, new aspects and contents are being added. In [4], there is a short definition of methodology as the doctrine, giving «knowledge about knowledge ... ways of obtaining the knowledge of the world». According to this, the methodology should contain the metaknowledge concept; the methodology of pedagogics - the concept of pedagogical metaknowledge, knowledge of pedagogical knowledge, its structure, knowledge of the subject; methodology of subject training - knowledge of methodical knowledge and knowledge of knowledge of a subject.

The educational sphere and its informational representation, educational process, informational educational interaction make the subject of modern pedagogics. Methodology of pedagogics is the doctrine about the logical structure of pedagogical activity. Identification of natural relations of its subject and the object of formation, principles of the processes organization of realization of methodological knowledge on the basis of these laws is supposed to take place. Since the truth is always circumstantial, the world knowledge development as well as methodological knowledge might be updated. That is, the processes of 
science knowledge developments and its methodology are interconnected and interconditioned. Thus the methodology of each differential pedagogical system should contain everything necessary and sufficient for functioning (formation, development, due application) of the educational system of corresponding level. Each level of pedagogical methodological metasystem requires the main methodological concept containing knowledge of:

- principles, means, general and main methods, conceptual features of this level:

- pedagogical categories, universal concepts, including, methodological;

- information concerning this level.

The methodological approach is determined as the basic direction of research, the point of view, «from which the object of study is considered as a concept or a principle leading the general strategy of research», [4]. According to I.L. Bim, «as a rule there's one initial idea at the heart of the approach» [2]. Since the strategy implies long-term objective (for example, victory) it is possible to conclude that the methodological approach is supposed to contain two components:

- A certain prospect, the ideal objective, its image;

- Principles of movement in the direction of this ideal purpose.

As a fundamental science, pedagogics realizes the system approach, which suggests the considering the investigated object as a single whole $\square$ a system which expresses the particular as the function of the general. The system approach in pedagogics assumes ordering and studying its subject via structure of its internal communications and relations. It assumes consideration of the object of research as a single whole systems, i.e., provides system representational ready in the first phase of research with the subsequent studying of particulars as components (functional displays) of the system. Thus any realization of it includes elements of intersystem communication. Therefore the system approach in its full expression and realization is system-intersystem, or metasystem one.

The metasystem approach is a new condition of the system approach supplemented with principles of intersystem interaction in global information metasystem. Hence, it is the basic methodological approach in pedagogics and is realized in all its scientific and pedagogical projections.

Under the influence of the global informatization, at the stage of formation and development of informational society the informational approach is as much significant methodological cognitive approach. Therefore the main methodological approach in science becomes system-informational, or the metasysteminformational approach, [5]. It is the expression of the system approach in the context of information representation and knowledge of environment, formation of an informational picture of the world, development of universal forms, ways and means of effective realization of these approaches.
System and information approaches assume mutual exploitation, being means of a mutual support and interaction:

- "System" indicates search and realization of system and intersystem communications, systematic character and systematization,

- "Informational" specifies the informational basis of knowledge - its environment and subjects, means and techniques, processes and relations, results and conclusions.

Systematic character means the systematical representation, determining relations, patterns, an invariant and variable condition, intersystem communication. It all has an abstract, hence, informational expression. Intersystem interaction is also expressed through the correlation of informational systems.

The informational approach assumes viewing the world through the prism of information processes and relations, interrelations and interferences of objects and the environment phenomena. Since the optimal condition of an environment object is the system, information interaction is intersystem.

Thus, the base methodological approach in pedagogics is the system-informational approach (metasystem) which is realized in education in system activity-based approach.

The system activity-based approach in education is realized through cognitive activity, activity development and education objects motivation, cognitive and developing interaction, synthesis and ordering of educational results (knowledge, abilities and skills).

Since each differential educational system has its own particular organization and the informationalcommunicational interaction peculiarities, the realization of the system-informational approach in it also assumes realization and the differentiated application of a number of other methodological approaches compatible with is system activity-based approach. Moreover, each such realization can have private or local characteristics, individual peculiarities of application with certain efficiency.

Realization of the system informational approach in education does not contradict, but assumes the application of the formal methodological approach on the differential basis: cognition of the general through the analysis of its contents, components and the subsequent synthesis, full or partial abstraction from the content.

The formal approach allows revealing the stable relations between the elements of the process or phenomenon in question. That is its appliance in research and teaching is based on the form or form support, defining and applying knowledge about it. It often receives a negative aspect as a manifestation of formalism. However, this is not true: the use of a formal approach is objectively necessary. But, at the same time it must be differentiated and be subject to the principles of the system approach. That is, if the system approach determines the strategy, then the formal one determines tactics, strategy implementation. Informational approach 
to knowledge also provides an abstraction and formalization, reliance on forms, communication, structure of informational relations.

Abstraction is a distraction from the specific properties of the original object, insignificant to this examination. This examination outlines the object, in a generalized form. Consequently, the abstract object is an object that summarizes the properties of a certain set of specific objects, but does not contain their specific properties. It is implemented in a certain set of concrete objects. An abstract object is perceived in two aspects as an objective factor and as an abstract concept.

Thus, in the process of examination (research, training) the abstraction allows the replacement of a particular object by the corresponding abstract concept, identified and endowed with semantic and other values. Then the knowledge obtained during this examination, is projected on the original objects. The higher the level of abstraction is, the higher the level of the abstract concepts and the higher the level of generalization.

An abstract object combines the image and the prototype of concrete objects, represented by it, model objects of the corresponding class and their free object. Identification of the object in the study or teaching system means its representation as a subject of study. Therefore, an abstract object is an object, represented by an abstract concept and its semantics. And since the subject involves its representation in its inherent forms, the abstraction indicates proper formalization:

- detection and description of all abstract forms,

- formalized expression of the object.

That is the formalization of the information (educational, etc) suggests its objective expression in its inherent form or representation in another form, which is more appropriate to the purposes and processing or storage of information. The form here is not only a means of studying the content of the information, but also an independent object of study.

Information exists as a combination of form and content. Therefore, scientific and educational information is studied in a balanced combination of formal and content-based methodological approaches.

Content-based approach deals with the study of the content of phenomena, information, processes, properties of the elements and their interaction, gaining knowledge about them with the help of abstraction, analysis of components of the content and synthesis of results and conclusions. Therefore, in the process of study, the content-based approach is implemented in combination with the formal approach, and the formal one in case it is not related to the content-based approach leads to formalization:

- The formal approach allows us to express contents properties of the object through the formal connections, interactions.

- The content-based approach allows us to study the form of the object through the manifestation of the content expressed by the form.

The formalization involves the study of the object in combination of its content and formal properties. Formal properties here are products of internal and external interactions of the object, the interaction of elements, components and external formal characteristics.

The content of the information is always concrete, while the form may be inherent in much information. This extends the possibilities of its study, makes it more universal, applicable to a wide field of information. Therefore, the use of the formal approach, the formalization is not only a necessary factor in a subjectbased teaching, but it also makes it more efficient and rational.

Studying the form of the object in isolation from its contents, reviewing or evaluation of the object of study only on the basis of the properties of its formal expressions devaluates them, leads to wrong results and conclusions. That is the formalism.

The formal approach is only self-sufficient in mathematics, algebra, where the abstraction of the content is absolute (limiting). However, mathematics also includes content-based approach: the harmony and aesthetic forms are gained; precise, mathematically correct means beautiful. Besides, each formal system has an internal interactive energy of its elements, components, which is included in the content. At the same time, modern scientific and applied research is more and more affected by the methods and forms of formal and analytical research. Universal informatization and mathematization of knowledge is in progress. This means that the formal approach, its principles and methods are entering the field of knowledge, differentiated scientific and educational areas in which informative approach prevails, in particular the field of humanities and educational subjects. Obviously, this should not be a goal in itself or a tribute to fashion.

Informatization of knowledge is:

1. Application of tools and products of informatization, IT industry, where knowledge of informatics is used.

2. Using the knowledge and techniques of informatics, reliance on formalization, modeling, classification in cognitive processes.

The mathematization of knowledge is the use of mathematical methods for analytical studies, use of methods and forms of algebraic representation, means of formal conclusions and implications, the structural analysis and construction.

- Mathematics is an analytical cognition language of formal and symbolic representation;

- Informatics is meta-language of science, the language of expressing the content through the form, meta-subject concepts and interactions.

Thus, the system-informational approach in education is associated with the formal and contentbased approaches, with formalization, synthesis and analysis, modeling, cross expressing of content and form. Vice versa, formalization involves formal representation of relations, communication and interaction. That is, the formalization leads to the systematization, structuring, and modeling. Hence, the concepts of the system, informational, formal, contentbased approaches are uniform. System-informational approach to knowledge is expressed by ways of formalization, structuring, organizing, system analysis, 
modeling, abstraction, mathematization, informatization and information presentation.

Socio-cultural approach to cognitive activities is aimed at the development of human society and culture, and assumes the realisation of all other approaches in this regard.

"The socio-cultural approach is a methodological approach based on the systematic approach, which considers society as an integrity that combines in it culture and sociality formed and transformed by human activity. This integrity, according to the principles of the system approach, forms a whole, the properties of which can't be inferred from the characteristics of the parts. The personality is considered as connected with the society by the system of relations and culture, combination of values and norms" [6].

The peculiarity of socio-cultural approach consists in the universality of its application in human world: every phenomenon, every element of its informational environment (information object, information process, interaction and attitude) have sociocultural characteristics. Therefore, they should be considered or implemented in the socio-cultural aspect in connection and interdependence with other phenomena and elements of the environment, i.e., in the aspect of the system approach. The socio-cultural aspect also has to consider a subject of cognitive activity, and its subject to subject and subject to object interaction with the informational environment. Therefore, socio-cultural methodological approach in education should be viewed upon as the social and cultural projection of a systematic approach.

Realization of the systematic approach in the sociocultural aspect turns the informational environment into a socio-cultural one, and the system approach into a methodological sociocultural system-informational approach.

Socio-cultural approach focuses not only on the social aspects of cognitive activity, but also on its environmental aspects, the aspects of informational security of the world and society, the preservation of unity with nature. It is also focused on opposition to the manifestations of formalism in the field of knowledge, technologizing human thinking, negative information and psychological influences.

The fundamental of the socio-cultural approach is focusing on the development of the informational world, personal information culture, moral and ethical values of the informational society.

Information culture characterizes the level of development of modern society in its transformation into informational one. It must manifest itself in each cognitive informational process, each informational interaction and express their relationship. That is, it must be reflected in every manifestation in the formation of socio-cultural and, therefore, socio-cultural informational system approach.

Foreign language teaching provides for the formation of socio-cultural competence of pupils as an expression of personal development of objects of teaching. Its formation and development is realized through learning the culture and intercultural relations of people - of native speakers and studied languages. It is natural in such teaching to rely on philological objects expressed in the literary, artistic form. These works have a high sociocultural and content, and a variety of expressive forms [7].

The linguistic forms and constructions (grammar, syntax) play a great role in foreign languages teaching. It may have a mathematical representation. Therefore, the methodological approach of modern teaching foreign languages is transformed into a social and cultural cognitive-communicative approach where formal, content-based, and socio-cultural approaches are expressed.

The cultural aspect, according to which information is considered as a component of human culture, a means of expression and the product of its manifestation, finds its expression in the socio-cultural approach to knowledge. It is a characteristic of a stage of human development and civilization, expressed in social, cultural, intellectual and spiritual values.

Culture is the highest level of the public domain. However, the state of society and, hence, its culture are characterized not only by spiritual values, but also by knowledge. It is also a public domain and a component of its general culture.

Knowledge and culture do not only determine the rest of the signs of the informational society, but they also determine each other:

- The development and application of knowledge requires the possession of the culture of knowledge and the culture of cognition.

- Informational culture is systematic and has a representation as a high-level knowledge, it develops and is realized through knowledge.

The culture of knowledge is the invariant abilities of an active system, of the knowledge and meta-knowledge carrier to effectively solve the informational and other problems; to create strong models of informational exchange; to create new knowledge on their basis. Culture of knowledge also includes the cognition culture, including personal cognition culture. This is knowledge about knowledge in its application to the field of knowledge, culture of knowledge perception and interaction with it.

Any knowledge is regular, and every unit of knowledge expresses certain regularity. Hence, the predicative formula $\left(\forall x_{1}^{i} \exists x_{i+1}^{n}\right)\left(\mathrm{F}\left(x_{1}^{n}\right)\right)$ corresponds to a unit of knowledge. However, the formal (predicative) verity of knowledge is only a necessary precondition, the rationale for its verity in the informational system, in which it finds its expression. Its specific realization may turn out to be false. Therefore, knowledge ought to be defined in the modal logic by modalities "necessary" and "possible."

Expanding the informational sphere requires changes in the education system, subjects, contents and forms of education, definition of information circulating in the field of education, communication tools and forms. Therefore, education should provide universal knowledge, form an outlook and culture of subjects, personal informational culture. The universalization of education and training subjects must gain. It is necessary 
to progress from data to knowledge and from the knowledge of the first level to the knowledge of higher levels, to structured knowledge.

Hence, the appropriate systematization and integration (according to the form and content) of educational material is required. It will increase the flexibility of learning and adaptation degree of the entity in his study environment. However, since a higher level of knowledge is the natural interaction of the knowledge of the previous level, it is necessary to rely on this knowledge.

Thus, there is no need for a large and haphazard multitude of data and knowledge of the previous level. Ordered sequences of elements, expressing this knowledge as natural interaction are required. Knowing these regularities, it is necessary to build the sequences, expressing them and appropriate sequences of teaching that knowledge. This will significantly simplify and facilitate the subject-based teaching.

The study of structured knowledge suggests access to interdisciplinary systems, reflected in the content of educational subjects. These systems and their pedagogical images are formed, for example, in the integrated field of mathematics and computer science, in multilingual teaching foreign languages, in the support of this teaching on mathematics, informatics, and information environment.

\section{References}

1. P.G. Schedrovitsky, By analyzing topics of organizational activity games (Pushchino, 1987) [In Rus]

2. I.L. Bim, Theory and practice of teaching the German language in high school (Moscow, Education 1988) [In Rus]

3. A.M. Novikov, and D.A. Novikov, World education education in the world, 1 (2008).

4. E.G. Yudin, Methodology of science. Consistency. Activities (Moscow, URSS, 1997) [In Rus]

5. J.G. Korotenkov, The philosophy of knowledge and information in the world. Monograph (Saarbrücken, Lap Lambert Academic Publishing 2014).

6. Socio-cultural approach. Available online: URL: http://ru.wikipedia.org/wiki (accessed on: 17.03.2015).

7. V.V. Korotenkova, Presentation of the content and methods of teaching a foreign language in terms of digital technology. Science of culture in the future (St. Petersburg «Digital Humanities», 2013). 